Frontiers of Information Technology \& Electronic Engineering

www.jzus.zju.edu.cn; engineering.cae.cn; www.springerlink.com

ISSN 2095-9184 (print); ISSN 2095-9230 (online)

E-mail: jzus@zju.edu.cn

\title{
Erratum:
}

\section{Erratum to: MDLB: a metadata dynamic load balancing mechanism based on reinforcement learning}

\author{
Zhao-qi WU $^{1}$, Jin $\mathrm{WEI}^{2,3}$, Fan ZHANG ${ }^{\dagger+1}$, Wei GUO ${ }^{1}$, Guang-wei XIE ${ }^{2,3}$ \\ ${ }^{I}$ National Digital Switching System Engineering \& Technological R\&D Center, Zhengzhou 450002, China \\ ${ }^{2}$ School of Computer Science, Fudan University, Shanghai 200433, China \\ ${ }^{3}$ Data Arena Institute, Fudan University, Shanghai 200433, China \\ †E-mail:17034203@qq.com
}

https://doi.org/10.1631/FITEE.19e0121

Erratum to: Front Inform Technol Electron Eng, 2020, 21(7):1034-1046

https://doi.org/10.1631/FITEE.1900121

Unfortunately the corresponding author's

ORCID was incorrect. It should be: Fan ZHANG,

https://orcid.org/0000-0001-7456-8377.

The online version of the original article can be found at https://doi.org/10.1631/FITEE.1900121

\footnotetext{
Corresponding author

(1) ORCID: Zhao-qi WU, https://orcid.org/0000-0001-7857-2875; Fan ZHANG, https://orcid.org/0000-0001-7456-8377

(C) Zhejiang University and Springer-Verlag GmbH Germany, part of Springer Nature 2020
} 